\title{
Cloud Computing-Positive Impacts and Challenges in Business Perspective
}

\author{
Aejaz Ahmad Dar* \\ School of Engineering and Technology, Islamic University of Science and Technology, Awantipora, Jammu and Kashmir, India
}

\begin{abstract}
Due to rapid growth of computing resources over the past years, cloud computing has been treated as a prominent research field and this technology appeared as a new solution in the IT field. Many businesses including small, medium and enterprise are migrating to this technology for so many reasons such as computing resources, reduced total cost of ownership, on demand services, increased revenue and many more. Cloud Computing offers number of advantages of cloud migration that motivated the business enterprises to adopt this change. There are so many challenges for the companies adopting cloud computing like interoperability, portability, organizational aspects and the most important challenge is the security and privacy of the information. There are different solutions or suggestions that a company should follow to overcome various challenges and issues.
\end{abstract}

Keywords: Cloud computing; Positive impact; Challenges; Solutions

\section{Introduction}

Cloud computing is the trending technology that companies decided to implement to grow in the market and achieve new business heights. In number of ways, we can see this new technology as an innovation [1]. T0068e investment in the real physical infrastructure in the business is much larger than the investment in the cloud. According to the statists portal, the total number of cloud-based users are approaching 3.4 billion, which is about half of the total population of the world in 2018. This statistical data clearly shows that the cloud computing technology is expanding exponentially, and it will soon dominate the information technology market. Because of the tremendous growth of this technology, every type of business should be well aware of the wave which is largely shaking the whole industry [2]. Cloud computing always has a great impact on the company. This technology not only impacts its global performances but also its internal organization especially IT sector. This technology is enhancing the usual methods of backing up of data. Cloud computing is bringing new methods and tools for the company that is using it [3].

Aim of present study is to highlight the positive impacts of cloud technology on business. The companies are facing some challenges and security issues during migration to this technology and there is a need to identify and discuss those challenges to have better planning and policy to overcome those issues $[4,5]$.

\section{Positive impacts of cloud computing on business organiza-} tion

Cloud computing have positive impact on the business organizations as it increases their revenue and helps them to achieve the business goals. Companies prefer to use the services offered by the cloud rather than building their own infrastructure. Following are the benefits of cloud computing technology which motivates the business organizations to migrate from local infrastructure to cloud (Figure 1).

Reduced cost: Cloud computing reduces the expenses of the company as the resources are only acquired when needed and only paid for when used as the billing model works as per usage and there is no up-front cost. The infrastructure is not purchased and thus lowering the initial expenses and maintenance cost as well. The clients are not the owners of the infrastructure but can use the cloud services online [6-9].

Unlimited scalability: This is the major benefit of cloud technology as the client has the flexibility to scale up or scale down as per the needs of the organization. The companies do not need to worry about the future demands as they can easily acquire the additional services anytime. Also, if a business grows over time, the cloud can scale effortlessly to meet the increased demand over time [10-13].

Flexibility: Cloud computing provides lot of flexibility to its clients. There is an easy testing and deployment of the services over cloud. The customers are free to decide which services they need and pay for accordingly. The cloud services can better meet the changing business demands by providing various services. If any application provided by the cloud is not getting our job done, we have the flexibility to switch to another cloud.

Better mobility: The users of the cloud can access the services of the cloud anytime anywhere from a variety of devices. Whenever they have the working internet connection, they can login and use the services. This benefit of cloud computing provides a flexible work culture to the employees and they can perform their duties from anywhere without the need to be physically present at the business headquarter.

Improved communication: Cloud computing improves communication and collaboration among employees by having access to instant messaging, conferencing and video conferencing options. They can jointly work on documents and projects ensuring higher cohesion and team work. This is possible because of data centralization and updation of cloud servers in real time.

Reliability: As the services of cloud is available all the time and can be accessed anywhere [14,15]. Also, the backup and recovery management make this technology more reliable.

Increased storage: Some cloud providers offer the Storage as service to its customers. Companies can store lot more data on cloud

*Corresponding author: Aejaz Ahmad Dar, School of Engineering and Technology Islamic University of Science and Technology, Awantipora, Jammu and Kashmir, India, Tel: 01933247954; E-mail: daraejaz@gmail.com

Received November 24, 2018; Accepted December 12, 2018; Published December 19, 2018

Citation: Dar AA (2018) Cloud Computing-Positive Impacts and Challenges in Business Perspective. J Comput Sci Syst Biol 12: 15-18. doi:10.4172/jcsb.1000294

Copyright: (c) 2018 Dar AA. This is an open-access article distributed under the terms of the Creative Commons Attribution License, which permits unrestricted use, distribution, and reproduction in any medium, provided the original author and source are credited. 
than their local devices. If business grows and demands more storage, companies effortlessly scale up and get more storage from the cloud provider [16,17].

Easier upgrades: It is the responsibility of the cloud providers to upgrade the infrastructure and services for their customers. The new business trends and solutions are made available to the clients by cloud providers to compete in the business market by adopting latest technologies. The cloud providers maintain the system by doing different software and security updates $[18,19]$.

Disaster recovery: Companies using cloud services need not to frame the complex disaster or failure recovery plans as the service providers take care of such issues and put the clients out of the trouble in a fast manner [12].

Security: The most important factor while choosing a cloud provider is the security and privacy they provide to our data. This is the main reason that these providers invest large amount on their services and infrastructure to offer better security.

\section{Open challenges in cloud migration}

When a company decides to migrate to cloud, it needs them to migrate their applications, sensitive data and other business elements from local devices to the cloud provider's site. The different challenges faced by the companies are (Figure 2).

Interoperability: Interoperability is the one of the big challenges in cloud migration. This is concerned with the ability of devices to communicate to each other. In cloud community technology, it means the ability to develop the code which works with number of cloud service providers simultaneously irrespective of the differences between the cloud providers. So, if we migrate the business to the cloud and want to be part of cloud environment, then it should be compatible with more than one service provider.

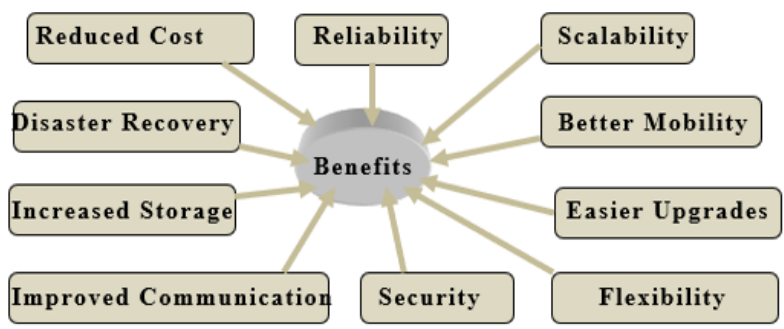

Figure 1: Benefits of Cloud Computing.

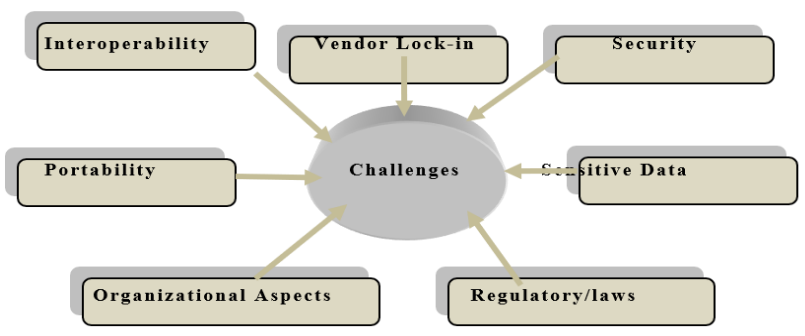

Figure 2: Cloud Computing Challenges.
Cloud security and privacy: Security of the cloud is the biggest threat in the current market. When we decide to adopt the cloud technology, we are migrating business sensitive information to the cloud servers and then there is less control over the data. It is then the responsibility of the cloud provider to ensure the data security and privacy. As the cloud services are accessed through internet, there is risk of attack by the hackers to steal in and cause the damage. It is, therefore, necessary to carefully choose the cloud provider and verify the methods and tools that are being used by the cloud to ensure the security and privacy to our data. Company should ensure some regulations and agreement with the cloud provider to enforce the data security.

Sensitive data: When these organizations adopt cloud computing, they need to migrate their sensitive data to cloud and if this data leaks, this causes big damage to the business. So, the migration of sensitive data to cloud is a big challenge and it needs great skills to handle and manage things.

Portability: Portability is the challenge in cloud computing which means that the applications should migrate easily from one cloud to another cloud. The software that we wish to move should be potable with the other cloud environment. This portability is difficult to achieve as the cloud providers use different standard languages for their respective platforms.

Vendor lock-in: It is a major barrier for most of the companies to migrate to cloud computing because of the lack of standardization. In vendor lock-in, customer wants to switch the vendor are bound to pay for changing the vendor. Vendor Lock-in is noticed in every technology till now despite number of efforts like open system and platform independent language.

Organizational aspects: The business organization adopting the cloud technology will undergo lot of changes in the internal working, mission, funding and staff etc. As the business is moving to the cloud, there will be new challenges for administration and the operational staff. There will be new security and privacy challenges as the data is stored on remote server. The employee of the company should be happy and confident of the new technology to explore the advantages and overcome the issues. The overall organizational setup will change and the employees at different levels should welcome this positive impact to achieve the new business heights.

Regulatory and laws restrictions: Before migrating to cloud, the business organization needs to keep in mind the different legal and regulatory issues. These laws ensure the commitment and responsibility of both clients and tenants. In few countries of the Europe, Government regulations cannot allow the personal data of customers or other sensitive information to be physically store outside country. The need to build exclusive centers may not be feasible for the cloud providers and may prove a big challenge for them. If sensitive data is not protected and some leakage occurs, then the defined laws and regulations will decide who is responsible for the fault and how to compensate.

\section{Solutions to overcome these challenges}

Despite number of benefits of cloud computing, there is low migration rate because of different challenges faced by the companies. Following are some solutions that a company should follow to overcome the challenges of cloud migration.

Use encryption technique: The best way to avoid data from snooping while sharing between business network and cloud devices is to encrypt it. The data is always transferred from local server to cloud 
server in an encrypted format so that even if it is hacked appears as trash to them.

Alternate backups: Companies should keep regular backups at their local storage devices or at the alternate cloud provider. There are so many cloud providers that are offering storage as a service to their clients.

Hiring skilled and experienced cloud professionals: As the companies are migrating their business to the cloud, there should be a team of skilled cloud professionals who know how to handle this technology efficiently. Hiring such team is foremost thing for a company who has sound knowledge about the technology and the standards used in technology.

Use the services of Security Service Providers: There are number of clouds provides in the market that is providing security as a service to their clients and company should use these services to ensure the security and privacy to their data.

Invest in education: Companies should educate their employees towards the goals of new technology in order to help learners to cope with the new adopted technology. Once the users get enough knowledge regarding positive impacts of the technology, they can render their full cooperation to make it a success in all respects [19].

Proper cloud provider selection: There are number of cloud service providers in the market who are competing and the company should select a service provider after proper investigation of the track record of the provider. Organization should verify the tools and techniques used by the cloud provider and choose the best one to meet their business requirement. Before migrating data to the cloud, the company should ask number of questions to the cloud provider regarding security and privacy measures taken, firewall setup, antivirus, testing methods etc. to ensure the data security and privacy threat in future.

Cloud auditor to audit the services: Third party control or organizations should be there to audit the cloud services and other performance including security. The Cloud Auditor should create an audit plan that includes policies and procedures as a reference guide.

Selective migration of business applications: company should first decide that which applications they need to migrate to cloud and which ones to keep on local devices. Before migration, they need to verify whether the application is finance based, is it customer data or business data because all the applications may not be cloud compatible.

Cloud service providers: Cloud Service providers are the companies which offer different cloud services to the clients like storage, databases, software, networking, servers etc. through the internet. These companies charge their clients for using the services as per usage (payas-you-go). There are number of cloud service providers competing in the market offering different services and giving users more or less control over their resources depending on the type of agreement. Cloud computing technology is offering services to the companies as Software as a service (SaaS), Platform as a service (PaaS) and Infrastructure as a service (PaaS). Software as a Service (SaaS) model provides hosted applications to its clients through internet. In Platform as Service (PaaS), a kind of development environment or software layer is provided as a service to the clients upon which other higher level of applications can be developed. Infrastructure as a Service provides servers, storage, networking infrastructure and computing capabilities as services via an internet connection (Figure 3).

In the current market there are number of cloud service providers like Amazon Web Services, Google Platform, Microsoft, HP, AT\&T etc. Also, more and more providers are emerging who develop their own applications or services upon infrastructure services provided by other market players (Table 1).

When we choose a Cloud provider, we must understand our business needs and the services offered by the provider. The user should sign the Service Level Agreement (SLA) after proper verification of the type of cloud and the services offered by the cloud. SLA defines the communication between service provider and the cloud consumer. This agreement helps in building user's trust in the cloud service provider.

\section{Conclusion}

Cloud computing is a modern technology trend that allows the companies to take over the market. Cloud computing is considered as an innovative and enhanced technology to run business organization. Most of businesses now-a-days are running all kinds of applications in the cloud like accounting, Custom built, communication, application development, productivity etc. Cloud based services are easy to afford for any kind of business as the customer need to pay only as per use.

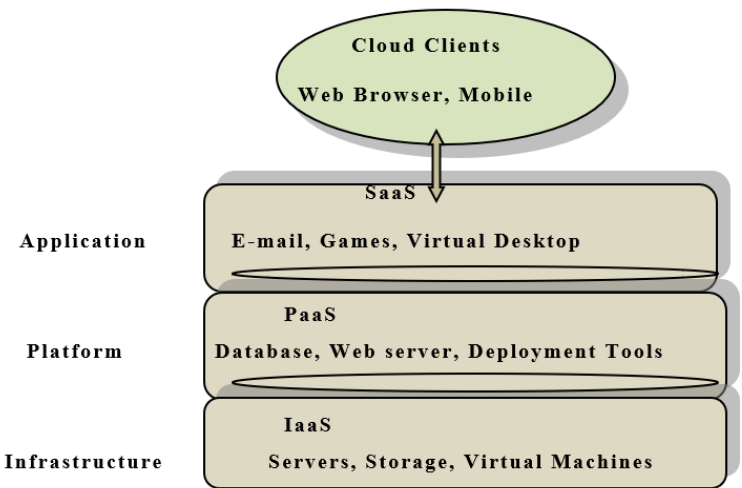

Figure 3: Cloud Service Models.

\begin{tabular}{|c|c|c|c|c|c|}
\hline Criteria & Amazon & Google & Microsoft & HP & AT\&T \\
\hline $\begin{array}{l}\text { Types of cloud } \\
\text { services provided }\end{array}$ & $\begin{array}{c}\text { laaS, PaaS, Storage, } \\
\text { Database }\end{array}$ & $\begin{array}{c}\text { laaS, SaaS, PaaS, Storage, Mobile, } \\
\text { Database, Big Data }\end{array}$ & $\begin{array}{l}\text { laaS, PaaS, Mobile, } \\
\text { Database, Big Data }\end{array}$ & $\begin{array}{l}\text { laaS, PaaS, Storage, } \\
\text { Database, DNS }\end{array}$ & $\begin{array}{c}\text { laaS, PaaS, Storage, } \\
\text { Network }\end{array}$ \\
\hline Key features & $\begin{array}{c}\text { Offering number of services } \\
\text { that are helping companies } \\
\text { to function better. }\end{array}$ & $\begin{array}{l}\text { Offers number of cloud computing } \\
\text { products via Google Apps, } \\
\text { Google compute Engine, Google } \\
\text { Cloud Storage, Big data, Mobile } \\
\text { Development Platform }\end{array}$ & $\begin{array}{l}\text { Media and Mobile } \\
\text { development }\end{array}$ & $\begin{array}{c}\text { Storage and cloud load } \\
\text { balancer, Open stack } \\
\text { software }\end{array}$ & $\begin{array}{c}\text { Providing a private } \\
\text { network for companies. }\end{array}$ \\
\hline Payment Plan & Pay per use, monthly & Pay per use & Pay per use, monthly, yearly & Pay per use & Pay per use \\
\hline
\end{tabular}

Table 1: Comparing Different Cloud Service Providers. 
Citation: Dar AA (2018) Cloud Computing-Positive Impacts and Challenges in Business Perspective. J Comput Sci Syst Biol 12: 15-18. doi:10.4172/ jcsb.1000294

Cloud providers offer more secure, more reliable and more scalable services to the customers. Cloud Providers periodically upgrade their services to offer better quality services to their clients to satisfy their business demands. Cloud computing is an easy way to cut down the company expenses and increase their revenue. The company using the cloud services has the benefit of accessing the information anytime anywhere and on any device. This has improved the decision-making capability and the productivity of the company has become faster and better. The different challenges thrown by adopting this technology can be reduced by using different encryption techniques, regular backups, proper selection of cloud provider etc.

\section{Acknowledgements}

The author extends they're thanks to the authorities of the of School of Engineering and Technology, Islamic University of Science and Technology, Awantipora, Jammu and Kashmir, for the facilities provided.

\section{References}

1. Stefanie L, Böhm M, Riedl C, Krcmar H (2010) The Business Perspective of Cloud Computing: Actors, Roles and Value Networks. p: 56.

2. Sundee BK (2018) Cloud Computing for Business. International Journal of Advances in Scientific Research and Engineering, p: 4.

3. Baciu El (2015) Advantages and disadvantages of cloud computing services, from the employee's point of view. SSRN 1: 95-101.

4. Buyya R, Vecchiola C, Thamarai SS (2013) Matering Cloud Computing: Foundation and application programming.

5. Lisievici A (2014) A few legal challenges related to the adoption of cloud-based solutions. Roman A Magazine of Business Law.
6. Bhopale SD (2013) Cloud Migration Benefits and Its Challenges Issue. IOSR Journal of Computer Engineering, pp: 40-45.

7. Iwona MW, Stefana $\mathrm{Cl}$ (2014) Cloud computing drives innovation.

8. Ankey J (2011) Heads in the cloud. Entrepreneur 39: 50-51.

9. THBS (2018) Cloud Computing Overview. Available from: https://www.thbs. com/thbs-insights/cloud-computing-overview.

10. Devasena LC (2014) Impact study of cloud computing on business development. Operations Research and Applications: An International Journal 1: 1-7.

11. Alexa H, Cebula J (2011) The basics of cloud computing. United States Computer.

12. Tutorials Point (2018) Cloud computing: cloud computing challenges. Available from: https://www.tutorialspoint.com/cloud_computing/cloud_computing challenges.htm

13. Martins O, Sahandi JR, Tian F (2016) Critical analysis of vendor lock-in and its impact on cloud computing migration: a business perspective. Journal of Cloud Computing 5: 1:4.

14. Tutorials Point (2018) Cloud computing security. Available from: https://www. tutorialspoint.com/cloud_computing/cloud_computing_security.htm

15. Holland R (2011) Ten Steps to Successful Cloud Migration.

16. Catalina SI (2015) Cloud computing - Impact on business.

17. Georgiou DA (2017) Security policies for cloud computing.

18. Agostino SD, Ahronovitz M, Armstrong J (2011) Moving to the Cloud (Version 1.0). A white paper produced by the Cloud Computing Use Cases Discussion Group.

19. Abdulelah A, Youssef AE (2014) Cloud Service Providers: A Comparative Study. International Journal of Computer Applications \& Information Technology, p: 5. 